\title{
Successful Invasion of a Food Web in a Chemostat*
}

\author{
GAIL S. K. WOLKOWICZ \\ Department of Mathematics and Statistics, McMaster University, \\ Hamilton, Ontario, Canada L8S $4 K I$
}

Received 10 March 1988; revised 12 August 1988

\begin{abstract}
A food web in a chemostat is considered in which an arbitrary number of competitor populations compete for a single, essential, nonreproducing, growth-limiting substrate, and an arbitrary number of predator populations prey on some or all of the competitor populations. Although any number of predator populations may prey on the same competitor population, each predator population preys on only one competitor population. The dynamics of substrate uptake is modeled by Lotka-Volterra or Michaelis-Menten (Holling type $\mathrm{I}$ or II), but the dynamics of competitor uptake is restricted to LotkaVolterra. Based on certain parameters, the model predicts the asymptotic survival or extinction of each of the different populations and suggests how competitor and/or predator populations could successfully invade the chemostat with or without causing a diverse ecosystem to crash. Similarly, it suggests how the elimination of certain populations could result in a more diverse or less diverse system.
\end{abstract}

\section{INTRODUCTION}

In this paper we consider a food web in a chemostat in which an arbitrary number of competitor populations compete for a single, essential, nonreproducing, growth-limiting substrate, and an arbitrary number of predator populations prey on some or all of the competitor populations. Although any number of predator populations may prey on the same competitor population, we assume that each predator population specializes exclusively in a single competitor population (see Figure 1).

In a chemostat, pure competition for a nonreproducing substrate results in the asymptotic survival of at most one competitor population even in the case of very general response functions (see, for example, Butler and Wolkowicz [4]). This paper addresses the problem of whether or not predation is one of the factors responsible for the diversity in ecosystems.

\footnotetext{
*This paper is dedicated to the memory of Professor Geoffrey J. Butler.
} 
Experimental evidence (e.g., Paine [17] and Slobodkin [21]) seems to indicate an affirmative response. Paine postulates that "Local species diversity is directly related to the efficiency with which predators prevent monopolization of the major environmental requisites by one species." He showed experimentally that the systematic removal of a single predator species from a specific ecosystem reduccd the number of species in the community from 15 to 8. My results also tend to support Paine's postulate. In addition, they predict which populations may be unable to invade an ecosystem and which may be able to successfully invade with or without causing a significant change in the number of other species in the system. They also suggest which populations can be eliminated without affecting the survival of the other species and which populations, if eliminated, could result in drastic changes in the size of the ecosystem. The criteria are based on the so-called break-even concentration of each species. For a competitor population this is the concentration of substrate at which that competitor population neither increases nor decreases, in the absence of predators and all other competitors. For a predator population it is the concentration of prey at which the predator population neither increases nor decreases in the absence of all other predator populations. Thus these break-even concentrations can be easily measured one species at a time before any competition takes place.

Certain assumptions with respect to the dynamics of the interactions allow a complete global analysis of the model. In particular, the competitors' dynamics are modeled by Lotka-Volterra or Michaelis-Menten responses and the predators' dynamics by Lotka-Volterra functional responses. As can be seen in Butler and Wolkowicz [5,6], allowing more general functional responses in the case of only two competitors and one predator population complicates the dynamics considerably (in particular, it allows more complicated types of invariant sets) even if we restrict ourselves to the class of monotone functional responses. I comment further on how allowing different functional responses affects the interpretation of the results in Section 4. In any case, there is still a basic message. One can conclude that tampering with just one species in our environment could conceivably lead to farreaching and drastic changes at many different levels that might be very far from what was expected. And these far-reaching changes could result from either the introduction of a species or the attempted elimination of a species.

This paper is organized in the following manner. In Section 2 the mathematical model of the food web to be studied is set down and the underlying assumptions are indicated. For convenience the model is scaled to produce an equivalent nondimensional version that is then analyzed in Section 3, where I first introduce notation for the critical points of this system and then state and prove the mathematical results. For those interested in the results but not in the related mathematics, this section can be omitted, since the results are restated in the discussion in Section 4, with 
their biological interpretation and an explanation of their ramifications. A technical lemma and its proof (used to prove the main result, Theorem 1), which concerns the possible invariant sets of Iotka-Volterra competitive systems coupled by a linear constraint, are given in the Appendix.

\section{THE MODEL}

The following model of predator-mediated competition in a chemostat is considered where $S(t)$ denotes the concentration of substrate as a function of time. There are $n$ competitor populations, indexed by the set $\Omega=$ $\{1,2, \ldots, n\}$, and $x_{i}(t), i \in \Omega$ denotes the concentration of the $i$ th competitor population as a function of time. Those competitor populations that have at least one predator population have their index in the set $\Upsilon$. Thus, if $k \in \Omega \backslash \Upsilon, x_{k}$ has no predators. If $k \in \Upsilon$, then $r_{k}$ denotes the number of different predator populations that prey on $x_{k}(t)$. The concentration of the $j$ th predator population on $x_{k}(t)$ is denoted by $y_{j}^{k}(t)$, where $k \in \Upsilon$ and $j \in\left\{1,2, \ldots, r_{k}\right\}$ (see Figures 1 and 2 ):

$$
\begin{aligned}
& \dot{S}(t)=\left[S^{0}-S(t)\right] D_{0}-\sum_{i \in \Omega} \frac{x_{i}(t) p_{i}(S(t))}{\eta_{i}} \\
& \dot{x}_{i}(t)=F_{i}\left(S(t), x_{i}(t), y_{1}^{i}(t), y_{2}^{i}(t), \ldots, y_{r_{i}}^{i}(t)\right) \triangleq F_{i}, \quad i \in \Omega \\
& \dot{y}_{j}^{i}(t)=y_{j}^{i}(t)\left[-\Delta_{j}^{i}+q_{j}^{i}\left(x_{i}(t)\right)\right], \quad i \in \Upsilon \text { and } j \in\left\{1,2, \ldots, r_{i}\right\}
\end{aligned}
$$

where

$$
\begin{aligned}
& F_{i}= \begin{cases}x_{i}(t)\left[-D_{i}+p_{i}(S(t))\right]-\sum_{j=1}^{r_{i} \frac{y_{j}^{i}(t) q_{j}^{i}\left(x_{i}(t)\right)}{\xi_{j}^{i}},} \quad \text { if } i \in \Upsilon \\
x_{i}(t)\left[-D_{i}+p_{i}(S(t))\right], & \text { if } i \in \Omega \backslash \Upsilon\end{cases} \\
& S(0) \geqslant 0, \quad x_{i}(0)>0 \quad i \in \Omega, \quad y_{j}^{i}(0)>0 \quad i \in \Upsilon, \quad j \in\left\{1,2, \ldots, r_{i}\right\}
\end{aligned}
$$

$r_{i}$ is a positive integer for each $i \in \Upsilon$, and $S^{0}, \eta_{i}, D_{i}, i \in \Omega$, and $\xi_{j}^{i}, \Delta_{j}^{i}, i \in \Upsilon$ and $j \in\left\{1,2, \ldots, r_{i}\right\}$, are positive constants. A dot over a symbol denotes the derivative with respect to time.

$S^{0}$ denotes the concentration of the substrate in the feed bottle, and $D_{0}$ represents the input rate from the feed bottle to the growth chamber and the washout rate from the growth chamber to the collecting bottle. (It is also referred to as the flow rate or dilution rate.) The $D_{i}$ and $\Delta_{j}^{i}$ each denote the sum of the dilution rate $D_{0}$ and the species-specific death rate.

The per capita growth rate of the $i$ th competitor as a function of substrate concentration is given by $p_{i}(S(t))$, which will be assumed to satisfy either Lotka-Volterra (i.e., Holling type I) or Michaelis-Menten (i.e., Holling type II) dynamics [see Holling [14] and Equations (8) and (9) below]. The 


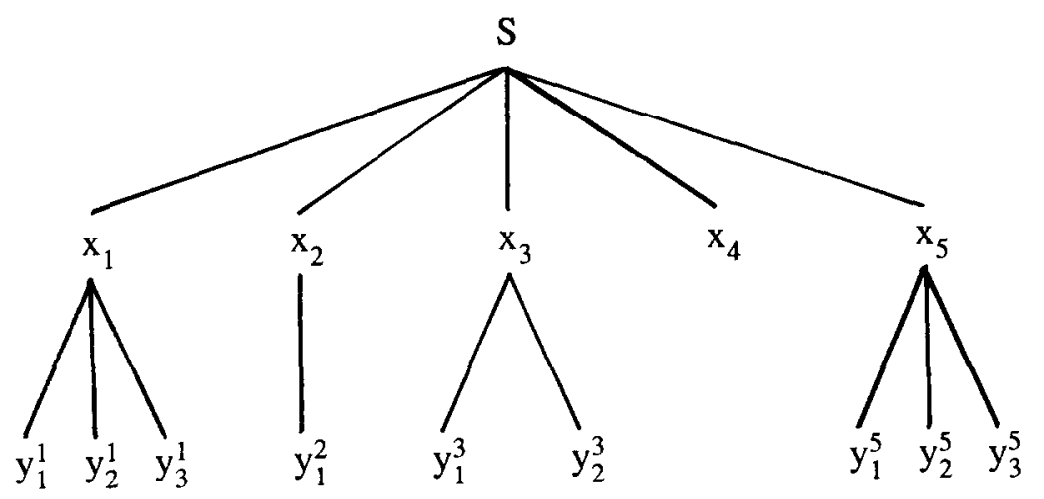

FIG. 1. The configuration of a sample food web. $\Omega=\{1,2,3,4,5\}$ and $\Upsilon=\Omega \backslash\{4\}$. $S$ denotes the substrate and $x_{k}$ the $k$ th competitor. $x_{k}$ has $r_{k}\left(r_{1}=3, r_{2}=1, r_{3}=2, r_{5}=3\right)$ predators, and $y_{j}^{k}$ denotes the $j$ th predator on $x_{k}$.

rate of consumption of substrate by the $i$ th competitor population is assumed to be proportional to the per capita growth rate, and so the $\eta_{i}$ are growth yield constants. Similarly, $q_{j}^{i}\left(x_{j}(t)\right)$ denotes the per capita growth rate of the $j$ th predator population $y_{j}^{i}(t)$ as a function of the concentration of the $i$ th competitor population $x_{i}(t)$, and the $\xi_{j}^{i}$ denote the corresponding growth yield constants. Thus we are allowing the predators to compete, but each predator specializes in one particular prey (competitor) population. The $q_{j}^{i}$ are assumed to be Lotka-Volterra.

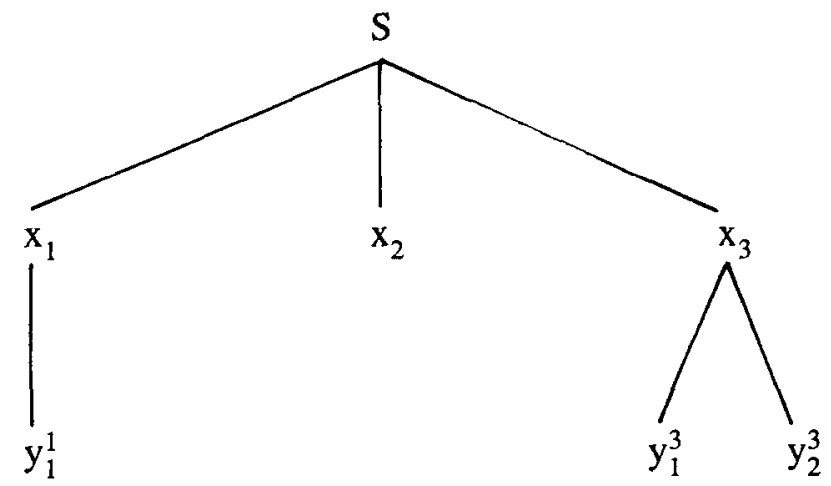

Fig. 2. The configuration of a sample food web. $\Omega=\{1,2,3\}$ and $\Upsilon=\{1,3\}$. $S$ denotes the substrate and $x_{k}$ the $k$ th competitor. $x_{k}$ has $r_{k}\left(r_{1}=1, r_{3}=2\right)$ predators, and $y_{j}^{k}$ denotes the $j$ th predator on $x_{k}$. 
It will be more convenient and there will be no loss of generality if we study a nondimensional version of the model. Let

$$
\begin{gathered}
\hat{S}=S / S^{0}, \quad \hat{x}_{i}=x_{i} /\left(\eta_{i} S^{0}\right), \quad \hat{y}_{j}^{i}=y_{j}^{i} /\left(\xi_{j}^{i} \eta_{i} S^{0}\right), \\
\hat{p}_{i}(\hat{S})=p_{i}(S) / D_{0}, \quad \hat{q}_{j}^{i}\left(\hat{x}_{i}\right)=q_{j}^{i}\left(x_{i}\right) / D_{0}, \\
\hat{D}_{i}=D_{i} / D_{0}, \quad \hat{\Delta}_{j}^{i}=\Delta_{j}^{i} / D_{0}, \quad \hat{t}=t D_{0} .
\end{gathered}
$$

With this scaling (omitting the hats, since this will not lead to any ambiguity), model (1)-(2) becomes

$$
\begin{aligned}
\dot{S}(t) & =1-S(t)-\sum_{i \in \Omega} x_{i}(t) p_{i}(S(t)) \\
\dot{x}_{i}(t) & =F_{i}\left(S(t), x_{i}(t), y_{1}^{i}(t), y_{2}^{i}(t), \ldots, y_{r_{i}}^{i}(t)\right) \triangleq F_{i}, \quad i \in \Omega \\
\dot{y}_{j}^{i}(t) & =y_{j}^{i}(t)\left[-\Delta_{j}^{i}+q_{j}^{i}\left(x_{i}(t)\right)\right], \quad i \in \Upsilon \text { and } j \in\left\{1,2, \ldots, r_{i}\right\},
\end{aligned}
$$

where

$$
F_{i}= \begin{cases}x_{i}(t)\left[-D_{i}+p_{i}(S(t))\right]-\sum_{j=1}^{r_{i}} y_{j}^{i}(t) q_{j}^{i}\left(x_{i}(t)\right), & \text { if } i \in \Upsilon \\ x_{i}(t)\left[-D_{i}+p_{i}(S(t))\right], & \text { if } i \in \Omega \backslash \Upsilon,\end{cases}
$$

$S(0) \geqslant 0, \quad x_{i}(0)>0, \quad i \in \Omega, \quad y_{j}^{i}(0)>0, \quad i \in \mathcal{T}, \quad j \in\left\{1,2, \ldots, r_{i}\right\}$.

Define $\lambda_{i}, i \in \Omega, \delta_{j}^{i}, i \in \Upsilon$ and $j \in\left\{1,2, \ldots, r_{i}\right\}$, so that

$$
p_{i}\left(\lambda_{i}\right)=D_{i} \quad \text { and } \quad q_{j}^{i}\left(\delta_{j}^{i}\right)=\Delta_{j}^{i} .
$$

Then $\lambda_{i}$ and $\delta_{j}^{i}$ are called the break-even concentrations of the nutrient. Assume that

$$
\lambda_{1}<\lambda_{2}<\cdots<\lambda_{n}
$$

and

$$
\delta_{1}^{i}<\delta_{2}^{i}<\cdots<\delta_{r_{i}}^{i} \text { for each } i \in \Upsilon \text {. }
$$

Since we are assuming that each $p_{i}(S(t))$ satisfies either Lotka-Volterra or Michaelis-Menten dynamics, it follows that $p_{i}$ has the form of either

$$
p_{i}(S)=D_{i} S / \lambda_{i} \quad \text { Lotka-Volterra }
$$


or

$$
p_{i}(S)=\frac{m_{i} D_{i} S}{\lambda_{i}\left(m_{i}-1\right)+S}, \quad m>1 \quad \text { Michaelis-Menten }
$$

This functional response is also referred to as Holling type II. [Actually, the general form for Michaelis-Menten dynamics is $p_{i}(S)=c_{i} S /\left(a_{i}+S\right)$, where $c_{i}$ and $a_{i}$ are positive constants. The constant $c_{i}$ is called the maximum growth rate and $a_{i}$ is called the half-saturation constant or Michaelis-Menten constant. In order for $\lambda_{i}>0$ to exist, $c_{i}$ must be greater than $D_{i}$. Since the break-even concentration plays such an important role in our results, it is more convenient and there is no loss of generality if we use the formulation given in (9) where $c_{i}=m_{i} D_{i}$ and so $m_{i}>1$ and $a_{i}=\lambda_{i}\left(m_{i}-1\right)$.]

Since $q_{j}^{i}\left(x_{i}\right)$ satisfies Lotka-Volterra dynamics,

$$
q_{j}^{i}\left(x_{i}\right)=\Delta_{j}^{i} x_{i} / \delta_{j}^{i} \text {. }
$$

If we modify (8) by specifying a maximum level $\bar{S}$ reached by $p_{i}(S)$, that is,

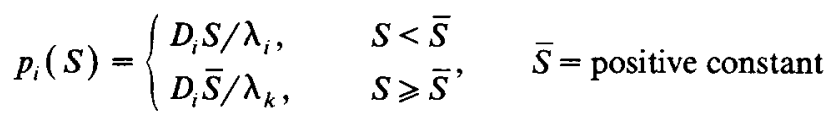

then $p_{i}(S)$ is referred to as Holling type I. For model (3) it can be shown that if $\bar{S}>1$, then there exists $T>0$ such that $S(t)<\bar{S}$ for all $t>T$. (The argument is similar to that given in [4, Theorem 3.1 and Corollary 4.1] using a differential inequality.) Thus all the results in this paper also apply if (8) is replaced by (11) provided $\bar{S}>1$. Similarly (10) can be modified:

$$
q_{j}^{i}\left(x_{i}\right)= \begin{cases}\Delta_{j}^{i} x_{i} / \delta_{j}^{i}, & x_{i}<\bar{x}_{i} \\ \Delta_{j}^{i} \bar{x}_{i} / \delta_{j}^{i}, & x_{i} \geqslant \bar{x}_{i}\end{cases}
$$

and the results apply if $\bar{x}_{i}>1$.

\section{ASYMPTOTIC PROPERTIES OF THE MODEL}

In this section we use a Lyapunov function approach to establish asymptotic properties, including local and global stability and persistence results, for model (3) (4).

We introduce the following notation for the critical points:

$$
\left(\bar{S}, \bar{x}_{1}, \bar{x}_{2}, \ldots, \bar{x}_{n}, \bar{y}_{1}^{i_{1}}, \bar{y}_{2}^{i_{1}}, \ldots, \bar{y}_{r_{1}}^{i_{1}}, \bar{y}_{1}^{i_{2}}, \ldots, \bar{y}_{r_{2}}^{i_{2}}, \ldots, \bar{y}_{1}^{i_{m}}, \ldots, \bar{y}_{r_{m}}^{i_{m}}\right)
$$

where $0 \leqslant m \leqslant n$ and for $j \in\{1,2, \ldots, m\}$

$$
i_{j} \in \Upsilon \triangleq\left\{i_{1}, i_{2}, \ldots, i_{m}\right\}, \quad i_{1}<i_{2}<\cdots<i_{m} .
$$

Recall that the index set $\Upsilon$ indicates which competitor populations have at least one predator population. Thus, in general, there are $n$ competitor 
populations but only $m$ of them have at least one predator. For example, in the food web in Figure 2 there are $n=3$ competitors, but only $m=2$ of them have at least one predator population. $\Upsilon=\left\{i_{1}, i_{2}\right\}=\{1,3\}$. Also, $r_{i j}$ indicates how many predators the $i_{j}$ th competitor has. In Figure 2, competitor $i_{1}=1$ has $r_{i_{1}}=r_{1}=1$ predators and competitor $i_{2}=3$ has $r_{i_{2}}=r_{3}=2$ predators. The critical points for this example, therefore, have the form $\left(\bar{S}, \bar{x}_{1}, \bar{x}_{2}, \bar{x}_{3}, \bar{y}_{1}^{1}, \bar{y}_{1}^{3}, \bar{y}_{2}^{3}\right\}$. The critical points in the food web in Figure 1 have the form $\left(\bar{S}, \bar{x}_{1}, \bar{x}_{2}, \bar{x}_{3}, \bar{x}_{4}, \bar{x}_{5}, \bar{y}_{1}^{1}, \bar{y}_{2}^{1}, \bar{y}_{3}^{1}, \bar{y}_{1}^{2}, \bar{y}_{1}^{3}, \bar{y}_{2}^{3}, \bar{y}_{1}^{5}, \bar{y}_{2}^{5}, \bar{y}_{3}^{5}\right\}$.

Model (3)-(4) only has two types of equilibria:

(i) Equilibria for which each competitor population with nonzero concentration has exactly one predator population with nonzero concentration, and

(ii) Equilibria for which each competitor population with nonzero concentration, except the one with the largest relative break-even concentration, has exactly one predator population, and the competitor population with the largest relative break-even concentration has no predator population with zero concentration.

The substrate concentration is always positive at equilibrium.

In order to distinguish each individual critical point we use the following notation. Equilibria of the form described in (i) will be denoted $E_{h_{1}, h_{2}, \ldots, h_{k}}^{j_{1}, j_{2}}$. The set of superscripts $\left\{j_{1}, j_{2}, \ldots, j_{k}\right\}$ indicates which competitors have nonzero concentration at equilibrium. The set of superscript-subscript pairs, $\left\{h_{1}, h_{2}, \ldots, j_{k}\right\}$, indicates which predators have nonzero concentration at the equilibrium. Similarly, critical points of the form described by (ii) will be denoted $E_{h_{1}, h_{2}, \ldots, j_{k-1}}^{j_{1}, j_{k}, j_{k}}$, indicating that competitors $j_{1}, j_{2}, \ldots, j_{k-1}$ each have a predator but competitor $j_{k}$ has no predator.

Remark 1. We define $i_{0}=0$. We use the convention that $E_{i_{0}}^{i_{0}}=E=$ $(1,0, \ldots, 0)$, the equilibrium at which all species die out and the substrate concentration equilibrates to 1 , and $E_{i_{0}}^{i_{1}}=E^{i_{1}}$, the equilibrium at which only the competitor population $i_{1}$ has nonzero concentration (and the substrate equilibrates to a positive value).

In the food web depicted in Figure 2 there are 11 different equilibria: $E$, $E^{1}, E^{2}, E^{3}, E_{1}^{1}, E_{1}^{3}, E_{2}^{3}, E_{1}^{1,2}, E_{1}^{1,3}, E_{1,1}^{1,3}$, and $E_{1,2}^{1,3}$. Here $E_{1,2}^{1,3}$ is of form (i). Competitors $x_{1}$ and $x_{3}$ have nonzero concentration, as do the corresponding predators $y_{1}^{1}$ and $y_{2}^{3}$. On the other hand, $E_{1}^{1,3}$ is of form (ii). Competitors $x_{1}$ and $x_{3}$ have nonzero concentrations, but only the predator $y_{1}^{1}$ corresponding to $x_{1}$ has nonzero concentration.

Next we specify exactly the actual equilibrium concentration of the substrate and of each competitor and predator population:

(i) For $h_{i} \in\left\{1,2, \cdots, r_{j_{i}}\right\}$, where $j_{1}<j_{2}<\cdots<j_{k}$ and $j_{i} \in \Upsilon$,

$$
E_{h_{1}, h_{2}, \ldots, h_{k}}^{j_{1}, j_{2}, \ldots, j_{k}}, \quad k \in\{0\} \cup \Omega
$$


satisfies $\bar{S}=\tilde{S}$, where $\tilde{S}$ is the unique solution of

$$
\begin{array}{ll} 
& 1-\tilde{S}=\sum_{i=1}^{k} \delta_{h_{i}}^{j_{i}} p_{j_{i}}(\tilde{S}) . \\
\bar{x}_{i}=0 \quad & \text { if } i \in \Omega \backslash\left\{j_{1}, j_{2}, \ldots, j_{k}\right\} \\
\bar{x}_{j_{i}}=\delta_{h_{i}}^{j_{i}} \quad & \text { if } i \in\{1,2, \ldots, k\} \\
\bar{y}_{h_{i}}^{j_{i}}=\delta_{h_{i}}^{j_{i}}\left(-D_{j_{i}}+p_{j_{i}}(\tilde{S})\right) / \Delta_{h_{i}}^{j_{i}} \quad \text { if } i \in\{1,2, \ldots, k\} \\
\bar{y}_{u_{i}}^{v_{z}}=0 \quad \text { if } v_{z} \notin\left\{j_{1}, j_{2}, \ldots, j_{k}\right\} \\
\quad \text { or if } v_{z} \in\left\{j_{1}, j_{2}, \ldots, j_{k}\right\}, \text { say, } v_{z}=j_{w}, \text { but } u_{i} \neq h_{w} ;
\end{array}
$$

(ii) $E_{h_{1}, h_{2}, \ldots, h_{k-1}}^{j_{1}, j_{2}, \ldots, j_{k-1}, j_{k}}$ for $k \in \Omega$ satisfies

$$
\begin{aligned}
\bar{S} & =\lambda_{j_{k}} \\
\bar{x}_{i} & = \begin{cases}0 & \text { if } i \in \Omega \backslash\left\{j_{1}, j_{2}, \ldots, j_{k}\right\} \\
\delta_{h_{i}}^{j_{i}} & \text { if } i \in\{1,2, \ldots, k-1\}\end{cases} \\
\bar{x}_{j_{k}} & =\left[1-\lambda_{j_{k}}-\sum_{i=1}^{k-1} \delta_{h_{i}}^{j_{i}} p_{j_{i}}\left(\lambda_{j_{k}}\right)\right] / D_{j_{k}} \\
\bar{y}_{h_{i}}^{j_{i}} & =\delta_{h_{i}}^{j_{i}} \frac{-D_{j_{i}}+p_{j_{i}}\left(\lambda_{j_{k}}\right)}{\Delta_{h_{i}}^{j_{i}}} \quad \text { if } i \in\{1,2, \ldots, k-1\} \\
\bar{y}_{u_{i}}^{v_{z}} & =0 \quad \begin{array}{l}
\text { if } v_{z} \notin\left\{j_{1}, j_{2}, \ldots, j_{k-1}\right\} \\
\text { or if } v_{z} \in\left\{j_{1}, j_{2}, \ldots, j_{k-1}\right\}, \text { say, } v_{z}=j_{w}, \text { but } u_{i} \neq h_{w} .
\end{array}
\end{aligned}
$$

\section{THEOREM I}

Consider model (3)-(4). Define $\lambda_{0}=0=\lambda_{n+1}$ and

$$
l=\max \left\{j \in \Upsilon: i_{j} \in \Upsilon \text { and } i_{j}=j\right\} .
$$

Then $0 \leqslant l \leqslant m$ [see (13)]. Exactly one of (i) and (ii) below holds:

(i) There exists a unique $h \in\{0,1,2, \ldots, l\}$ such that

$$
1-\lambda_{h}-\sum_{i=1}^{h} \delta_{1}^{i} p_{i}\left(\lambda_{h}\right)>0
$$

and

$$
1-\lambda_{h+1}-\sum_{i=1}^{h} \delta_{1}^{i} p_{i}\left(\lambda_{h+1}\right)<0
$$


in which case

(a) $E_{1,1, \ldots, h}^{1,2, \ldots, h}$ is stable, and all other critical points are unstable.

(b) $S \rightarrow \tilde{S}$, where $\tilde{S}$ is the unique solution of

$$
1-\tilde{S}=\sum_{i=1}^{h} \delta_{1}^{i} p_{i}(\tilde{S})
$$

and satisfies

$$
\lambda_{h}<\tilde{S}<\lambda_{h+1}
$$

$$
x_{i} \rightarrow 0 \text { if } i \in \Omega \backslash\{1,2, \ldots, h\} \quad \text { and } \quad y_{j}^{i} \rightarrow 0 \text { if } i \notin\{1,2, \ldots, h\} \text { or } j \neq 1 \text {. }
$$

(c) If $h \in\{0,1,2\}$, then $E_{1,1, \ldots, 1}^{1,2, \ldots, h}$ is globally asymptotically stable.

(d) If $h \in\{1,2, \ldots, l\}$, then

$$
\lim _{t \rightarrow \infty} x_{i}(t)>0 \text { if } i \in\{1,2, \ldots, h\} \text { and } \underset{t \rightarrow \infty}{\lim } y_{1}^{i}(t)>0 \text { if } i \in\{1,2, \ldots, h\}
$$

(i.e., the first $h$ competitors and the first $h$ corresponding predators persist).

(ii) There exists a unique $h \in\{1,2, \ldots, l\}$ such that

$$
1-\lambda_{h}-\sum_{i=1}^{h-1} \delta_{1}^{i} p_{i}\left(\lambda_{h}\right)>0
$$

and

$$
1-\lambda_{h}-\sum_{i=1}^{h} \delta_{1}^{i} p_{i}\left(\lambda_{h}\right)<0
$$

or

$$
h=l+1
$$

and

$$
1-\lambda_{h}-\sum_{i=1}^{h-1} \delta_{1}^{i} p_{i}\left(\lambda_{h}\right)>0
$$

in which case

(a) $E_{1,1, \ldots, 1}^{1,2, \ldots, 1, h}$ is stable, and all other critical points are unstable,

(b) $S \rightarrow \lambda_{h}, x_{i} \rightarrow 0$ if $\mathrm{i} \in \Omega \backslash\{1,2, \ldots, h\}$, and $y_{j}^{i} \rightarrow 0$ if $i \notin\{1,2, \ldots, h-1\}$ or $j \neq 1$.

(c) If $h \in\{1,2,3\}$, then $E_{1,1, \ldots, 1}^{1,2, \ldots, h-1, h}$ is globally asymptotically stable. 
(d) If $h \in\{1,2, \cdots l, l+1\}$, then

$$
\lim _{t \rightarrow \infty} x_{i}(t)>0 \text { if } i \in\{1,2, \ldots, h\}
$$

and

$$
\varliminf_{t \rightarrow \infty} y_{1}^{i}(t)>0 \text { if } i \in\{1,2, \ldots, h-1\}
$$

(i.e., the first $h$ competitors and the first $h-1$ corresponding predators persist).

Proof. Let

$$
\theta \triangleq\{1,2, \ldots, h\}
$$

(i)(a) Define the Lyapunov function

$$
\begin{aligned}
V= & V\left(S, x_{1}, x_{2}, \ldots, x_{n}, y_{1}^{i_{1}}, y_{2}^{i_{1}}, \ldots, y_{r_{1}}^{i_{1}}, y_{1}^{i_{2}}, \ldots, y_{r_{i_{2}}}^{i_{2}}, \ldots, y_{1}^{i_{m}}, \ldots, y_{r_{i_{m}}}^{i_{m}}\right) \\
= & S-\tilde{S}-\tilde{S} \ln (S / \tilde{S})+\sum_{i \in \Theta} k_{i}\left[x_{i}-\delta_{1}^{i}-\delta_{1}^{i} \ln \left(\frac{x_{i}}{\delta_{1}^{i}}\right)\right]+\sum_{i \in \Omega \backslash \Theta} k_{i} x_{i} \\
& +\sum_{i \in \Theta} k_{i}\left[y_{1}^{i}-\tilde{y}_{1}^{i}-\tilde{y}_{1}^{i} \ln \left(\frac{y_{1}^{i}}{\tilde{y}_{1}^{i}}\right)\right]+\sum_{i \in \Theta} \sum_{j=2}^{r_{i}} k_{i} y_{j}^{i}+\sum_{i \in \Upsilon \backslash \Theta} \sum_{j=1}^{r_{i}} k_{i} y_{j}^{i}
\end{aligned}
$$

where $\tilde{y}_{1}^{i}=\delta_{1}^{i}\left[-D_{i}+p_{i}(\tilde{S})\right] / \Delta_{1}^{i}$ if $i \in \Theta$ and if $p_{i}$ is Lotka-Volterra, $k_{i}=1$, but if $p_{i}$ is Michaelis-Menten, then

$$
k_{i}= \begin{cases}\frac{\left(m_{i}-1\right) \lambda_{i}+\tilde{S}}{\lambda_{i}\left(m_{i}-1\right)}, & i \in \Theta \\ \frac{m_{i}}{m_{i}-1}, & i \in \Omega \backslash \Theta\end{cases}
$$

Note that (15)-(17) imply that

$$
\lambda_{h}<\tilde{S}<\lambda_{h+1} .
$$

Then after algebraic manipulation and the observation that the $k_{i}, i \in \Omega$, were chosen so that

$$
(\tilde{S}-S) \frac{p_{i}(S)}{S}+k_{i}\left[-D_{i}+p_{i}(S)-\frac{\Delta_{1}^{i} \tilde{y}_{1}^{i}}{\delta_{1}^{i}}\right]=0, \quad i \in \Theta
$$

and

$$
(\tilde{S}-S) \frac{p_{i}(S)}{S}+k_{i}\left[-D_{i}+p_{i}(S)\right] \leqslant 0, \quad i \in \Omega \backslash \Theta
$$


[In fact (27) ensures that the coefficient of $x_{i}$ in $\dot{V}$ vanishes for each $i \in \Theta$, and (28) ensures that for each $i \in \Omega \backslash \Theta$ the coefficient of $x_{i}$ is nonpositive. The constant $k_{i}$ needed for (27) to hold can be found only if $p_{i}$ satisfies Lotka-Volterra or Michaelis-Menten dynamics.] It follows that

$$
\begin{aligned}
& \dot{V}=(S-\tilde{S}) \frac{(1-S)}{S}+\sum_{i \in \Theta} x_{i}\left\{k_{i}\left[-D_{i}+p_{i}(S)\right]+p_{i}(S) \frac{\tilde{S}-S}{S}\right\} \\
& +\sum_{i \in \Omega \backslash \Theta} x_{i}\left\{k_{i}\left(-D_{i}+p_{i}(S)\right)+p_{i}(S) \frac{\tilde{S}-S}{S}\right\} \\
& -\sum_{i \in \Theta} k_{i} \delta_{1}^{i}\left[-D_{i}+p_{i}(S)\right]-\sum_{i \in \Theta} \sum_{j=1}^{r_{i}} k_{i} x_{i} \frac{\Delta_{j}^{i} y_{j}^{i}}{\delta_{j}^{i}} \\
& +\sum_{i \in \Theta} \sum_{j=1}^{r_{i}} k_{i} \delta_{1}^{i} \frac{\Delta_{j}^{i} y_{j}^{i}}{\delta_{j}^{i}} \\
& +\sum_{i \in \Theta} k_{i} \Delta_{1}^{i}\left[\tilde{y}_{1}^{i}-y_{1}^{i}+y_{1}^{i} \frac{x_{i}}{\delta_{1}^{i}}-\tilde{y}_{1}^{i} \frac{x_{i}}{\delta_{1}^{i}}\right] \\
& +\sum_{i \in \Theta} \sum_{j=2}^{r_{i}} k_{i} \Delta_{j}^{i} y_{j}^{i} \frac{x_{i}-\delta_{j}^{i}}{\delta_{j}^{i}}-\sum_{i \in \Upsilon \backslash \Theta} \sum_{j=1}^{r_{i}} k_{i} \Delta_{j}^{i} y_{j}^{i} \\
& =(S-\tilde{S}) \frac{1-S}{S}+\sum_{i \in \Theta} k_{i} \delta_{1}^{i}\left[p_{i}(\tilde{S})-p_{i}(S)\right] \\
& +\sum_{i \in \boldsymbol{\Theta}} x_{i}\left\{k_{i}\left[-D_{i}+p_{i}(S)-\tilde{y}_{1}^{i} \frac{\Delta_{1}^{i}}{\delta_{1}^{i}}\right]+p_{i}(S) \frac{\tilde{S}-S}{S}\right\} \\
& +\sum_{i \in \Omega \backslash \Theta} x_{i}\left\{k_{i}\left[-D_{i}+p_{i}(S)\right]+p_{i}(S) \frac{\tilde{S}-S}{S}\right\} \\
& +\sum_{i \in \Theta} \sum_{j=2}^{r_{i}} k_{i} \Delta_{j}^{i} y_{j}^{i} \frac{\delta_{1}^{i}-\delta_{j}^{i}}{\delta_{j}^{i}}-\sum_{i \in \Upsilon \backslash \Theta} \sum_{j=1}^{r_{i}} k_{i} \Delta_{j}^{i} y_{j}^{i} \\
& =(S-\tilde{S}) \frac{1-S-\sum_{i \in \Theta} \delta_{1}^{i} p_{i}(S)}{S}+\sum_{i \in \Omega \backslash \Theta} x_{i} p_{i}(S) \frac{\tilde{S}-\lambda_{i}}{S} \\
& +\sum_{i \in \Theta} \sum_{j=2}^{r_{i}} k_{i} \Delta_{j}^{i} y_{j}^{i} \frac{\delta_{1}^{i}-\delta_{j}^{i}}{\delta_{j}^{i}}-\sum_{i \in \Upsilon \backslash \Theta} \sum_{j=1}^{r_{i}} k_{i} \Delta_{j}^{i}, y_{j}^{i} \\
& \leqslant 0 \quad \text { by (17) and (26). }
\end{aligned}
$$

Therefore $E_{1,1, \ldots, 1}^{1,2, \ldots h}$ is stable.

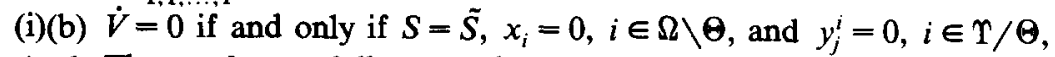
or $j \neq 1$. The result now follows by the LaSalle extension theorem (see Hale [13]). 
(i)(c) If $h \in\{0,1\}$, then it is easy to see that the only invariant set in

$$
\mathscr{A}=\left\{\left(S, x_{1}, \ldots, x_{n}, y_{1}^{i_{1}}, \ldots, y_{r_{1}}^{i_{1}}, \ldots, y_{1}^{i_{m}}, \ldots, y_{r_{i_{m}}}^{i_{m}}\right) \in \mathbf{R}_{+}^{n+m+1}: \dot{V}-0\right\}
$$

(where $\mathbf{R}_{+}^{n+m+1}$ is the $(n+m+1)$-dimensional nonnegative cone) is the equilibrium solution, that is, $E$ if $h=0$ and $E_{1}^{1}$ if $h=1$. If $h=2$, any invariant subset of $\mathscr{A}$ must satisfy

$$
\begin{aligned}
& \dot{x}_{i}(t)=x_{i}(t)\left[-D_{i}+p_{i}(\tilde{S})-y_{1}^{i}(t) \Delta_{1}^{i} / \delta_{1}^{i}\right], \quad i=1,2 \\
& \dot{y}_{1}^{i}(t)=y_{1}^{i}(t)\left[-\Delta_{1}^{i}+x_{i}(t) \Delta_{1}^{i} / \delta_{1}^{i}\right], \quad i=1,2
\end{aligned}
$$

and since $S \equiv \tilde{S}$ (where $\lambda_{1}<\tilde{S}<\lambda_{2}$ ), $\dot{S}=0$, and so

$$
x_{1}(t) p_{1}(\tilde{S})+x_{2}(t) p_{2}(\tilde{S})=1-\tilde{S} \quad \text { for all } t \geq 0 .
$$

If we let $\alpha_{i}=-D_{i}+p_{i}(\tilde{S}), \sigma_{i}=\Delta_{1}^{i}, \beta_{i}=\Delta_{1}^{i} / \delta_{1}^{i}, \quad \gamma_{i}=p_{i}(\tilde{S}), i=1,2$, and $W=1-\tilde{S}$, then (30) and (31) take the form of system (A.1) of Lemma A.1 (see Appendix), and so it follows that the only invariant subset of $\mathscr{A}$ is the equilibrium solution $E_{1,1}^{1,2}$. Again the result follows by the LaSalle extension theorem.

(i)(d) If $h=1$ or 2, the result follows from (c) above. If $h=3$, by (b), $x_{i} \rightarrow 0$ if $i=4, \ldots, n$ and $y_{j}^{i} \rightarrow 0$ if $i \geqslant 4$ or if $j \neq 1$. Also, $S \rightarrow \tilde{S}$, where $\tilde{S}$ is the unique solution of

$$
1-\tilde{S}=\sum_{i=1}^{3} \delta_{1}^{i} p_{i}(\tilde{S})
$$

and $\lambda_{3}<\tilde{S}<\lambda_{4}$. Therefore any invariant subsets of $\mathscr{A}$ [see (29)] must satisfy

$$
\begin{aligned}
& \dot{x}_{i}(t)=x_{i}(t)\left(-D_{i}+p_{i}(\tilde{S})-y_{1}^{i}(t) \Delta_{1}^{i} / \delta_{1}^{i}\right), \quad i=1,2,3 \\
& \dot{y}_{1}^{i}(t)=y_{1}^{i}(t)\left(-\Delta_{1}^{i}+x_{i}(t) \Delta_{1}^{i} / \delta_{1}^{i}\right), \quad i=1,2,3
\end{aligned}
$$

and

$$
\sum_{i=1}^{3} x_{i}(t) p_{i}(\tilde{S})=1-\tilde{S} \quad \text { for all } t \geqslant 0
$$

These are three simultaneous, independent Lotka-Volterra predator-prey systems coupled only the condition that a certain linear combination of the $x_{i}$ is constant. Therefore for each $i \in\{1,2,3\}$, either $\left(x_{i}(t), y_{i}(t)\right) \rightarrow(0,0)$ or $\left(x_{i}(t), y_{i}(t)\right)$ converges to the positive equilibrium or $\left(x_{i}(t), y_{i}(t)\right)$ is a 
periodic solution with $x_{i}(t)>0$ and $y_{i}(t)>0$ for all $t>0$. Therefore it suffices to show that $\left(x_{i}(t), y_{i}(t)\right)$ does not converge to $(0,0)$ for any $i \in\{1,2,3\}$. If it did, by Lemma A.1 and (i)(c) we would obtain a contradiction to (32). For $h>3$ the result follows by a straightforward induction argument.

(ii)(a) Define the Lyapunov function

$$
\begin{aligned}
& V=V\left(S, x_{1}, x_{2}, \ldots, x_{n}, y_{1}^{i_{1}}, y_{2}^{i_{1}}, \ldots, y_{r_{1}}^{i_{1}}, y_{1}^{i_{2}}, \ldots, y_{r_{2}}^{i_{2}}, \ldots, y_{1}^{i_{m}}, \ldots, y_{r_{i_{m}}}^{i_{m}}\right) \\
& =S-\lambda_{h}-\lambda_{h} \ln \left(\frac{S}{\lambda_{h}}\right)+\sum_{i \in \Theta} k_{i}\left[x_{i}-\bar{x}_{i}-\bar{x}_{i} \ln \left(\frac{x_{i}}{\bar{x}_{i}}\right)\right]+\sum_{i \in \Omega \backslash \Theta} k_{i} x_{i} \\
& +\sum_{i \in \Theta \backslash\{h\}} k_{i}\left[y_{1}^{i}-\bar{y}_{1}^{i}-\bar{y}_{1}^{i} \ln \left(\frac{y_{1}^{i}}{\bar{y}_{1}^{i}}\right)\right] \\
& +\sum_{i \in \Theta \backslash\{h\}} \sum_{j=2}^{r_{i}} k_{i} y_{j}^{i}+\sum_{i \in \Upsilon \backslash(\boldsymbol{Q} \backslash\{h\})} \sum_{j=1}^{r_{i}} k_{i} y_{j}^{i}
\end{aligned}
$$

where

$$
\begin{gathered}
\bar{x}_{i}=\delta_{1}^{i}, i \in \Theta /\{h\}, \quad \bar{x}_{h}=\frac{1-\lambda_{h}-\sum_{i \in \Theta \backslash \backslash h\}} \delta_{1}^{i} p_{i}\left(\lambda_{h}\right)}{D_{h}} \\
\bar{y}_{1}^{i}=\delta_{1}^{i} \frac{-D_{i}+p_{i}\left(\lambda_{h}\right)}{\Delta_{1}^{i}} \quad \text { if } i \in \Theta \backslash\{h\}
\end{gathered}
$$

and if $p_{i}$ is Lotka-Volterra, $k_{i}=1$, but if $p_{i}$ is Michaelis-Menten, then

$$
k_{i}= \begin{cases}\frac{\left(m_{i}-1\right) \lambda_{i}+\lambda_{h}}{\lambda_{i}\left(m_{i}-1\right)}, & i \in \Theta \\ m_{i} /\left(m_{i}-1\right), & i \in \Omega \backslash \Theta\end{cases}
$$

It follows [as in (i)(a)] that

$$
\begin{aligned}
\dot{V}= & \left(S-\lambda_{h}\right) \frac{1-S-\sum_{i \in \Theta} \bar{x}_{i} p_{i}(S)}{S}+\frac{\sum_{i \in \Omega \backslash \Theta} x_{i} p_{i}(S)\left(\lambda_{h}-\lambda_{i}\right)}{S} \\
& +\sum_{i \in 1}^{h-1} \sum_{j=2}^{r_{i}} k_{i} \Delta_{j}^{i} y_{j}^{i} \frac{\delta_{1}^{i}-\delta_{j}^{i}}{\delta_{j}^{i}}-\sum_{i \in T \backslash \Theta} \sum_{j=1}^{r_{i}} k_{i} \Delta_{j}^{i} y_{j}^{i} \\
& +\chi_{\Upsilon}(h) \sum_{j=1}^{r_{h}} k_{h} \Delta_{j}^{h} y_{j}^{h} \frac{\bar{x}_{h}-\delta_{j}^{h}}{\delta_{j}^{h}}
\end{aligned}
$$

$\leqslant 0 \quad$ by the definition of $\bar{x}_{h}$ and by (19) or (22), 
where

$$
\chi_{\Upsilon}(h)= \begin{cases}1 & \text { if } h \in \Upsilon \\ 0 & \text { if } h \notin \Upsilon\end{cases}
$$

That $E_{1,1, \ldots, 1}^{1,2, \ldots, h} 1, h$ is stable follows since $V$ is a Lyapunov function.

(ii)(b), (c), and (d). The proof is similar to (i)(b), (c), and (d), above, provided one notes that in the case $h=3$, any invariant set in $\mathscr{A}$ must satisfy $S \equiv \lambda_{3}$ and $y_{3} \equiv 0$, which implies that $\dot{x}_{3} \equiv 0$ and so $x_{3}$ is constant.

Remark 2. I conjecture that Lemma A.1 is true for any positive integer $k$. If this is so, then the global asymptotic stability of the equilibrium solution in Theorem 1 (i)(c) and (ii)(c) would hold without the restrictions on $h$.

\section{SUMMARY AND DISCUSSION}

We begin this section by summarizing the results of Section 3 in a less technical manner. First note that the model allows only two types of equilibria:

(i) Equilibria for which each competitor species with nonzero concentration has exactly one predator species with nonzero concentration, and

(ii) Equilibria for which each competitor species with nonzero concentration, except the one with the largest relative break-even concentration, has exactly one predator species. The one with the largest relative break-even concentration has no predator species with nonzero concentration.

(At equilibrium, the concentration of substrate is always positive.)

Second, there is always exactly one asymptotically stable equilibrium. (All other equilibria are unstable.) Which equilibrium is stable is determined by the values of the break-even concentrations of the predators and the prey [see (15)-(16) and (19)-(22)]. The smaller the break-even concentrations, the better the chance that more species will have nonzero concentration at the asymptotically stable equilibrium. In all cases, if the stable equilibrium has exactly $h$ competitor species with nonzero concentration, then they are the first $h$ competitor species (i.e., the $h$ competitor species with the lowest break-even concentrations, $\lambda_{i}$ ). The single surviving predator species for each of these competitors is the associated predator species with the lowest break-even concentration, $\delta_{1}^{i}$. (I shall henceforth refer to this associated predator species as the best predator for that particular competitor species.)

Third, if the stable equilibrium contains at most two competitor species with nonzero concentration (and hence at most two predator species with nonzero concentration) or if it contains three competitor species with nonzero concentration and two predator species with nonzero concentration, then it 
is globally asymptotically stable with respect to the interior of the positive cone. In fact, it seems likely that the stable equilibrium is always globally asymptotically stable; however, I was not able to prove this. In any case, if it is not globally asymptotically stable, then the only other possibilities for attractors are periodic orbits in which exactly the same populations are represented with nonzero concentration as in the stable equilibrium. Thus the model predicts that if a population has positive concentration in the stable equilibrium, then that population always persists (in the mathematical sense defined by Butler and Waltman [2] (i.e., its $\underline{\varliminf}_{t \rightarrow \infty}$ is positive), which is purely deterministic and does not take into consideration the possibility of extinction due to stochastic effects as in Stephanopoulos and Fredrickson [22]), whereas if it has zero concentration in the stable equilibrium then it eventually dies out, regardless of the initial (positive) concentrations of the populations.

As far as the effects of invasion by or attempted elimination of species, one can make the following predictions based on the analysis of the model in Section 3:

(i) A predator-prey pair can successfully invade a food web (where we assume the invading predator species preys solely on the invading prey species) if the corresponding break-even concentrations, say $\delta^{*}$ and $\lambda^{*}$ of the predator and prey, respectively, are sufficiently small. For example, if a food web is at equilibrium or its populations are predicted to approach an equilibrium or periodic orbit at which precisely the first $h$ competitors persist, and either

$$
\lambda_{h}<\lambda^{*} \quad \text { and } \quad 1-\lambda^{*}-\sum_{i=1}^{h} \delta_{1}^{i} p_{i}\left(\lambda^{*}\right)-\delta^{*} p^{*}\left(\lambda^{*}\right)>0
$$

or there exists an integer $k$ such that

$$
\lambda_{k}<\lambda^{*}<\lambda_{k+1} \leqslant \lambda_{h} \quad \text { and } \quad 1-\lambda^{*}-\sum_{i=1}^{k} \delta_{1}^{i} p_{i}\left(\lambda^{*}\right)-\delta^{*} p^{*}\left(\lambda^{*}\right)>0
$$

where $p^{*}$ denotes the response function of the invading competitor, then the invasion will be successful (i.e., the invading predator-prey pair will persist). Whether or not the invasion is successful and whether or not it results in the extinction of other populations depends on how the inequalities in (15)-(16) and (19)-(22) are affected. The only species that could be driven to extinction due to the invasion are competitor populations with break-even concentrations larger than $\lambda^{*}$ and/or the associated predator populations. The model predicts that invasion by a predator-prey pair will not further increase the diversity by resulting in the persistence of any other new populations. 
(ii) The model predicts that a predator species alone can successfully invade provided there is an appropriate prey (competitor) population at nonzero concentration at the time of the invasion already in the food web, the invading predator species is the best predator associated with that prey, and the break-even concentrations of the invading predator and the already existing prey are sufficiently small. As in case (i), how small depends on the inequalities in (15)-(16) and (19)-(22). Invasion by a predator can actually result in a significant increase in the number of species persisting. For example, if before an invasion there is a food web in which $x_{1}$ has no predator, then regardless of initial conditions all the populations of predators and competitors would eventually approach extinction except possibly $x_{1}$. If, however, the invading predator preys on $x_{1}$ and competitor populations $x_{1}-x_{5}$ all have relatively small break-even concentrations and the associated predators also have relatively small break-even concentrations, in particular, if

$$
1-\lambda_{5}-\sum_{i=1}^{5} \delta_{1}^{i} p_{i}\left(\lambda_{5}\right)>0
$$

then the model predicts that the invasion will result in $x_{1}-x_{5}$ and their associated best predators all persisting. Similar reasoning suggests how systematic elimination of a predator could result in the collapse of an ecosystem. This certainly adds support to the claim of many ecologists that predation is one of the factors responsible for the diversity in natural ecosystems. It appears that in this model, predator-mediated coexistence works because a predator limits a given competitor, say $x_{i}$, and prevents it from holding substrate concentration at too low a level $\lambda_{i}$. This allows some competitors with $\lambda$ such that $\lambda_{i}<\lambda$ to coexist with $x_{i}$. Of course, predation is not the only mechanism for obtaining coexistence. For example, self-regulation by competitors or interference competition can also lead to coexistence of several competitors.

(iii) A competitor population alone could successfully invade a food web provided its break-even concentration, say $\lambda^{*}$, is sufficiently small. More precisely, if the stable equilibrium contains $h$ nonzero competitor populations and $\lambda^{*}<\lambda_{h}$, then the invading competitor will persist. This could lead to a significant collapse in the food web since the invading competitor population has no predators. All previously persisting competitor populations with break-even concentration larger than $\lambda^{*}$ and their associated predators would now be driven to extinction except possibly one competitor population. Again, using the same reasoning, the model predicts that systematic elimination of a competitor population could result in a significant increase in the diversity of the ecosystem. 
One must be careful not to take the results too literally. The predictions obtained were derived from a mathematical model in which the prey response to substrate density was modeled by either Lotka-Volterra or Michaelis-Menten dynamics and predator response to prey density was modeled by Lotka-Volterra dynamics. Unfortunately, these assumptions were for technical reasons. Although for many simple microorganism populations Michaelis-Menten is a good model of the dynamics, it is unlikely that Lotka-Volterra would be a good model of the predator dynamics.

In Butler and Wolkowicz [5] a model involving at most two competitors competing for substrate and a predator preying on the best competitor was studied. General monotone response functions were allowed. One of the main differences between their results and ours is that in their model there is the possibility of a Hopf bifurcation. Also it may be the case that if the break-even concentrations are sufficiently small none of the equilibria in their model is stable. On the other hand, although they did not prove it, in all the examples they considered, if some equilibrium was not a global attractor there was a globally attracting periodic orbit. And just as in my model, which species survived and which were driven to extinction depended on the relative values of the break-even concentrations. In fact they proved that provided all the break-even concentrations were sufficiently small, all populations in their model persisted uniformly (although not necessarily at equilibrium).

In Butler and Wolkowicz [6] a model involving three competitors competing for substrate and a predator preying on the best competitor was considered. Again, general monotone dynamics were used to model the species response functions. Just as in this model they found that if any competitor persisted one of them was always the competitor with the lowest break-even concentration and a second competitor was able to persist only provided the predator was able to persist. However, they found that the second survivor was not necessarily the one with the second lowest break-even concentration. Instead, the second survivor depended on the initial concentrations of the species.

Cohen [8] found that in the food webs he surveyed the number of species of predator typically exceeded the number of species of prey by an average of 1.3 predator species per prey species. This model seems to indicate that if each predator species preys on only a single prey species, each prey species will ultimately (asymptotically) have at most one surviving predator species. It might be enough to allow more general predator response functions as in Butler and Wolkowicz [5] to account for this discrepancy in predator-prey ratio. Clearly, more modeling must be done using more general response functions before the situation can be completely understood.

Modeling of a similar food web in which the predators are allowed to prey on more than one competitor would also be of interest. This, of course, 
requires modeling the predator strategy for consumption of prey. For example, does the predator just consume whatever prey it can catch, or does it have a preference for one particular prey species and only consume the other when the preferred species falls below some threshold?

Shigesada et al. [20] consider a classical Lotka-Volterra $n$-species competition model (i.e., they do not explicitly model the substrate) in which they consider the effects of interference competition and derive criteria for the invasion of a new species. Understanding the effect of allowing interference competition in the chemostat model studied in this paper would also be useful.

There is a vast amount of research, both theoretical and experimental, on related submodels of the model analyzed here. In particular, the food chain that consists of the substrate, one consumer, and its predator or the food web that consists of one substrate, two competitors, and a predator has been studied in [1], [3], [5], [7], [9]-[12], [15], [16], [18], [19], [22], and [23], just to mention a few sources. The articles by Fredrickson [10] and Butler and Wolkowicz [5] describe many of the contributions.

\section{APPENDIX}

\section{LEMMA A.I}

Consider the simultaneous Lotka-Volterra predator-prey systems

$$
\begin{array}{cc}
\dot{x}_{i}(t)=x_{i}(t)\left(\alpha_{i}-\beta_{i} y_{i}(t)\right), & i \in\{1, \ldots, k\} \\
\dot{y}_{i}(t)=y_{i}(t)\left(-\sigma_{i}+\beta_{i} x_{i}(t)\right), & i \in\{1, \ldots, k\} \\
\sum_{i=1}^{k} \gamma_{i} x_{i}(t)=W \quad & \text { for all } t \geqslant 0 \\
x_{i}(0)>0, \quad y_{i}(0)>0, \quad i \in\{1, \ldots, k\}
\end{array}
$$

where $k \in\{1,2\}$ and $\gamma_{i} i \in\{1, \ldots, k\}$ and $W$ are positive constants. Then

(i) $W=\sum_{i=1}^{k} \gamma_{i} \sigma_{i} / \beta_{i}$, and

(ii) The only solution of (A.1) is the positive equilibrium solution

$$
\left(\sigma_{1} / \beta_{1}, \ldots, \sigma_{k} / \beta_{k}, \alpha_{1} / \beta_{1}, \ldots, \alpha_{k} / \beta_{k}\right) .
$$

Proof. If $k=1$ the result is obvious. Assume that $k=2$.

(i) Since $\gamma_{1} x_{1}(t)=W-\gamma_{2} x_{2}(t)$, it follows that $x_{1}$ and $x_{2}$ are periodic with the same minimum period, say $T$. Assume $T>0$, or there is nothing to prove. $T$ is finite since for each $i$ the phase portrait of each Lotka-Volterra predator-prey system is a center. Now, for each $i \in\{1,2\}$,

$$
0=\int_{0}^{T} \frac{\dot{y}_{t}(t)}{y_{i}(t)} d t=\int_{0}^{T}\left[-\sigma_{i}+\beta_{i} x_{i}(t)\right] d t .
$$


This implies that

$$
\frac{1}{T} \int_{0}^{T} x_{i}(t) d t=\frac{\sigma_{i}}{\beta_{i}}
$$

But then

$$
W=\frac{1}{T} \int_{0}^{T} W d t=\frac{1}{T} \int_{0}^{T} \sum_{i=1}^{2} \gamma_{i} x_{i}(t) d t=\sum_{i=1}^{2} \gamma_{i} \frac{\sigma_{i}}{\beta_{i}}
$$

(ii) As in (i) we may assume that $x_{1}$ and $x_{2}$ have the same finite minimum period $T>0$. (Then $y_{1}$ and $y_{2}$ each also have the same period $T$.) There exists $\tau \geqslant 0$ such that $x_{1}(\tau)=\sigma_{1} / \beta_{1}$. Since $\gamma_{1} \ddot{x}_{1}(t)=-\gamma_{2} \ddot{x}_{2}(t)$ for all $t \geqslant 0$

$$
\gamma_{1} x_{1}\left(\alpha_{1}-\beta_{1} y_{1}\right)^{2}-\beta_{1} x_{1} \dot{y}_{1}=-\gamma_{2} x_{2}\left(\alpha_{2}-\beta_{2} y_{2}\right)^{2}+\beta_{2} x_{2} \dot{y}_{2} \quad \text { for all } t \geqslant 0 \text {. }
$$

But $x_{1}(\tau)=\sigma_{1} / \beta_{1}$ implies that $x_{2}(\tau)=\sigma_{2} / \beta_{2}$, and so $\dot{y}_{1}(\tau)=0=\dot{y}_{2}(\tau)$. But then $y_{i}(\tau)=\alpha_{i} / \beta_{i}, i \in\{1,2\}$. This implies that we are at equilibrium, contradicting the assumption that the minimum period $T>0$. The result follows immediately.

Research partially supported by Natural Science and Engineering Research Council of Canada grant A9358 and by a Science and Engineering Research Board grant from McMaster University.

\section{REFERENCES}

1 H. R. Bungay and M. L. Bungay, Microbial interactions in continuous culture, $A d v$. Appl. Microbiol. 10:269-312 (1968).

2 G. J. Butler and P. Waltman, Persistence in dynamical systems, J. Differ. Equations 63:255-263 (1986).

3 G. J. Butler, S. B. Hsu, and P. Waltman, Coexistence of competing predators in a chemostat, J. Math. Biol. 17:133-151 (1983).

4 G. J. Butler and G. S. K. Wolkowicz, A mathematical model of the chemostat with a general class of functions describing nutrient uptake, SIAMJ. Appl. Math. 45:138-151 (1985).

5 G. J. Butler and G. S. K. Wolkowicz, Predator-mediated competition in a chemostat, J. Math. Biol. 24:167-191 (1986).

6 G. J. Butler and G. S. K. Wolkowicz, Predator-mediated coexistence in a chemostat: coexistence and competition reversal, Math. Modelling Sci. Technol. 8:781-785 (1987).

7 R. P. Canale, An analysis of models describing predator-prey interaction, Biotech. Bioeng. 17:353-378 (1970).

8 J. E. Cohen, Food Webs and Niche Space, Princeton University Press, Princeton, New Jersey, 1978.

9 J. F. Drake and H. M. Tsuchiya, Predation of Escherichia coli by Colpoda stenil, Appl. Environ. Microbiol. 31:870-874 (1976). 
10 A. G. Fredrickson, Behavior of mixed cultures of microorganisms, Ann. Rev. Microbiol. 31:63-87 (1977).

11 T. C. Gard, Persistence in fond chains with general interactions, Math. Biosci. 51:165-174 (1980).

12 T. C. Gard, Mathematics analysis of some resource-prey-predator models: application to a NPZ microcosm model, in Population Biology Proceedings, Edmonton, H. I. Freedman and C. Strobeck, eds., Lecture Notes in Biomath. Vol. 52, Springer, New York, 1982.

13 J. K. Hale, Ordinary Differential Equations, Wiley-Interscience, New York, 1969.

14 C. S. Holling, The functional response of predators to prey density and its role in mimicry and population regulation, Mem. Entomol. Soc. Canada 45:3-60 (1965).

15 J. L. Jost, J. F. Drake, H. M. Tsuchiya, and A. G. Fredrickson, Microbial food chains and food webs, J. Theor. Biol. 41:461-484 (1973).

16 J. L. Jost, J. F. Drake, A. G. Fredrickson, and H. M. Tsuchiya, Interactions of Tetrahymena pyriformis, Escherichia coli, Azotobacter vinelandii, and glucose in a minimal medium, J. Bacteriol. 113:834-840 (1973).

17 R. T. Paine, Intertidal community structure: experimental studies on the relationship between a dominant competitor and its principal predator, Oecologia 15:93-120 (1974).

18 P. T. Saunders and M. J. Bazin, On the stability of food chains, J. Theor. Biol. 52:121-142 (1975).

19 G. R. Sell, What is a dynamical system? in Studies in Ordinary Differential Equations, J. K. Hale, ed., MAA Series in Mathematics, Vol. 14, 1977, pp. 32-51.

20 N. Shigesada, K. Kawasaki, and E. Teramoto, The effects of interference competition on stability, structure and invasion of a multi-species system, J. Math. Biol. 21:97-113 (1984).

21 L. B. Slobodkin, Ecological populations of Hydrida, J. Anim. Ecol. 33(Suppl.): 131-148 (1964).

22 G. Stephanopoulos and A. G. Fredrickson, Extinction probabilities in microbial predation: a birth and death approach, Bull. Math. Biol. 43:165-181 (1981).

23 H. M. Tsuchiya, J. F. Drake, J. L. Jost, and A. G. Fredrickson, Predator-prey interaction of Dictyostelium discordeum and Escherichia coli in continuous culture, J. Bacteriol. 110:1147-1153 (1972). 